\title{
LAS NUEVAS DIMENSIONES DEL DERECHO HUMANO A LA SALUD. CUIDADOS PALIATIVOS.
}

\begin{abstract}
RESUMEN. Las nuevas dimensiones del derecho humano a la salud dentro de las cuales se encuentran los cuidados paliativos constituyen un importante desafío para los Estados en el marco de las obligaciones asumidas en materia sanitaria. Los cuidados paliativos ponen su mirada central en la persona, deben proporcionarse en forma interdisciplinaria a través de servicios de salud integrados en todos los niveles asistenciales, atendiendo enfermedades limitantes, crónicas o incurables que lo demanden, y ser proporcionados a pacientes de todas las edades atendiendo su entorno y allegados. Obstáculos de diversa naturaleza se identifican al momento de hacer efectivo el goce del derecho a los cuidados paliativos. Entre algunos de ellos, se encuentran la insuficiencia o falta de recursos humanos especializados y recursos materiales adecuados que aseguren la cobertura integral a nivel país así como también entre otras barreras, la insuficiente información a la población sobre el alcance de esta dimensión del derecho a la salud y el derecho a acceder a los mismos.
\end{abstract}

PALABRAS CLAVES. Salud. Cuidados paliativos. Dolor. Derechos Humanos. Acceso a la salud.

ABSTRACT. New dimensions of fundamental human right to health, including palliative care, represent an important challenge for States within the framework of obligations undertaken regarding health issues. Palliative care focus on the person, must be interdisciplinary rendered by means of comprehensive health services in all levels of care, must serve limiting, chronic and incurable diseases as may be required, and must be provided to patients covering the entire age range. Constraints of diverse

* Doctora en Derecho y Ciencias Sociales. Profesora (agr.) de Derechos Humanos y Bioética en la Facultad de Derecho UDELAR. Profesora de Derechos Humanos y Bioética en la Facultad de Derecho CLAEH. Diploma Superior en Bioética (FLACSO). Presidenta de la Institución Nacional de Derechos Humanos y Defensoría del Pueblo. Autora de diversos libros en su especialidad entre ellos "Manual de Derechos Humanos"; "Derecho al reconocimiento de la dignidad humana"; "Derechos Humanos en movimiento". Correo electrónico: ius@netgate.com.uy 
nature may be identified upon exercising the right to palliative care, including but not limited to deficiency or lack of specialized human resources and appropriate material resources at country level, as well as lack of appropriate information to the population with regards to the scope of this dimension of the right to health and the right to access thereof.

KEY WORDS. Health. Palliative Care. Pain. Human Rights. Access to Health.

\section{EL DERECHO HUMANO A LA SALUD COMO DERECHO INCLUSIVO}

El derecho a la salud integra el grupo de derechos de naturaleza económica y social. Su inclusión en esta categoría se justifica en tanto requiere de la actuación activa y progresiva del Estado para satisfacer las demandas de atención sanitaria de la población a través de la asignación de recursos económicos. En función de dicha particularidad en relación a la protección del goce del derecho se puede considerar que el derecho al acceso a la salud forma parte de las obligaciones del Estado como forma de satisfacer el valor de la salud como derecho inherente a la persona humana.

Del punto de vista conceptual, el derecho a la salud es pre condición para la vida de los seres humanos. Se erige a partir del reconocimiento del valor de la dignidad humana. No puede definirse como un derecho incondicional a estar sano, pues la salud depende de diversos factores que quedan fuera del control directo del Estado como la estructura biológica de cada ser humano y las condiciones en las cuales se habita. En consecuencia se entiende que el derecho a la salud, consiste en "el acceso al nivel más alto de posible de salud física y mental" lo que implica el "derecho a disfrutar de un conjunto de bienes instalaciones, servicios y condiciones" necesarios para su realización. ${ }^{1}$

Su carácter "inclusivo" comprende los factores determinantes básicos de la salud como disponer de agua potable, saneamiento, condiciones sanitarias adecuadas, alimentos aptos para el consumo, nutrición y viviendas adecuadas, condiciones de trabajo y medio ambiente salubre, educación e información en cuestiones de salud e igualdad de género. ${ }^{2}$

La construcción del concepto de salud, originariamente incorporado en textos de máxima jerarquía constitucional sin mayores especificaciones ha ido profundizándose en función de los principios de igualdad y no discriminación. El derecho a acceder al nivel más alto de salud física y mental comprende todos los niveles de atención, tanto el preventivo como el de atención clínica o tratamiento, así como también post tratamiento. Con el transcurso del tiempo se han desarrollado aspectos de la salud que incorporan lo sexual y reproductivo, habiéndose legislado a tales efectos como una de las prestaciones sanitarias

\footnotetext{
Puede ampliarse en OMS ONU. "El derecho a la salud". Folleto informativo No. 31. Ginebra, 2008.

2 Ilustra significativamente este concepto ZOBOLI al señalar: "La salud y la enfermedad provienen del modo de vivir de las personas, incluyendo su inserción en los momentos de producción y reproducción social. Mas que un estado es un proceso salud enfermedad, determinado por factores históricos y sociales constituyentes y determinantes" ZOBOLI, Elma. "Salud integral". En Diccionario Latinoamericano de Bioética. (Juan Carlos Tealdi Coordinador). Editado por UNESCO, RedBioética y la Universidad Nacional de Colombia. Colombia, 2009, pp. 565 y ss.
} 
y sin distinción etárea lo que implicó una nueva demanda de los usuarios y pacientes en relación al clásico modelo de atención sanitaria. ${ }^{3}$

Todo este proceso determina que el derecho de los habitantes a acceder al más alto nivel de vida posible, demande a los Estados la instrumentación de políticas públicas que aseguren la igualdad de oportunidades a la población; la prevención en su amplia perspectiva así como el tratamiento de patologías y enfermedades de todo tipo incluyendo las limitantes y crónicas; la obtención de medicamentos y tratamientos esenciales que las permitan enfrentar o paliar los efectos que las patologías puedan tener para los pacientes en su vida cotidiana; la educación e información a nivel generalizado; además de la necesaria participación de la población en el proceso de adopción de decisiones en cuestiones relacionadas con la salud a nivel comunitario y nacional.

El desarrollo del derecho humano a la salud unido a la irrupción de las nuevas tecnologías en el ámbito sanitario, han puesto de manifiesto la necesidad de proteger el vínculo con la libertad y auto determinación de la persona también en el ámbito sanitario lo que irrumpe en una realidad social que basada en el respeto a los derechos humanos exige una mirada integral de la condición del individuo. El reconocimiento y respeto a la libertad en el ámbito sanitario comprende una constante evolución en aras de fortalecer la auto determinación del sujeto en relación a las decisiones sanitarias que le incumben. El consentimiento "libre e informado" de la persona tanto en la etapa de prevención, diagnóstico o terapéutica e incluso en relación a las decisiones que vinculan al final de sus días, órganos, células y tejidos, constituye un requisito sustancial para asegurar la protección del derecho a la autonomía. Esto supone el reconocimiento de la capacidad y necesidad de consentir tratamientos, medicamentos, prácticas y todo tipo de decisiones en relación al cuerpo. Esta evolución conlleva un cambio de paradigma en la "relación paciente - equipo asistencial", proceso que apunta a sustituir el modelo "paternalista" a otro basado en la "autonomía", reconociendo en los individuos la capacidad y necesidad de asumir las decisiones que vinculan a su propia condición humana lo que no implica desconocer la existencia de límites en función del interés general o decisiones que afecten a terceros. Entre diversos aspectos puede verse el avance en relación al reconocimiento del derecho a no ser sometido a investigaciones médicas sin consentimiento de la persona respetando el principio de autonomía. Así también el derecho a no ser sometido a esterilizaciones forzadas o cualquier tipo de violencia sexual o de otra índole. ${ }^{4}$

\section{CONSAGRACIÓN NORMATIVA FUENTE NACIONAL E INTERNACIONAL}

El derecho a la salud está reconocido en la gran mayoría de las constituciones nacionales tanto en países desarrollados como otros que no alcanzan tales niveles de desarrollo. ${ }^{5}$ Dentro

3 La ley 18426 de diciembre 2008 "Defensa del derecho a la salud sexual y reproductiva”, estableció la obligación del Estado de garantizar condiciones para el ejercicio pleno de los derechos sexuales y reproductivos de toda la población. A tales efectos deberá promover "políticas nacionales de salud sexual y reproductiva, diseñará programas y organizará servicios para desarrollarlos". Disponible en: https:/ /legislativo.parlamento.gub.uy/temporales/leytemp3968148.htm Fecha de consulta: 10 agosto 2019.

4 El Pacto de Derechos Civiles y Políticos de 1966 (ONU) establece en su artículo 7: "Nadie será sometido a torturas ni a tratos crueles, inhumanos o degradantes. En particular nadie será sometido sin su libre consentimiento a experimentos médicos o científicos”. 
de éstas algunas fórmulas incluyen el derecho a la salud y el deber de cuidar y atender la propia salud como derechos inherentes a la persona humana. En materia legislativa se verifican diferentes previsiones sobre la salud como derecho humano. Leyes que consagran "derechos y obligaciones de pacientes y usuarios" (es el caso de Uruguay con la aprobación de la ley 18335 en 2008) con perspectivas que profundizan las nuevas dimensiones sanitarias y otras previsiones más específicas como ser las relativas a la "donación y trasplante de órganos, células y tejidos"; "voluntades anticipadas"; "reproducción asistida"; "derechos sexuales y reproductivos" ya mencionada. El proceso apunta a introducir especificaciones más concretas referidas a la condición de salud, promoviendo en algunos casos su consagración con jerarquía constitucional.

Uruguay introdujo la protección de la salud en la reforma de la Constitución de $1934 .{ }^{6}$ El artículo 44 de la Constitución establece la obligación del Estado de procurar el perfeccionamiento físico, moral y social de todos los habitantes lo que supone el reconocimiento implícito del derecho humano a la salud que comprende los aspectos médicos, éticos y sociales. Desde la mirada inclusiva que referimos anteriormente, la interpretación del texto constitucional nos lleva a vincularlo con el derecho a la vida, la integridad física, el entorno vital, la igualdad y la libertad lo que se proyecta a través de los artículos 7, 8, 10, 47 y 72 . La norma constitucional prevé el "deber de cuidar de la propia salud" y "asistirse en caso de enfermedad" lo que correlativamente puede entenderse tal como enseña CASSINELLI MUÑOZ, como el derecho a cumplir con los deberes que la Constitución establece. ${ }^{7}$ Ante este derecho - deber, el tercer inciso del mismo artículo 44 dispone que el Estado proporcionará gratuitamente los medios de prevención y de asistencia tan sólo a los "indigentes o quienes carecen de recursos suficientes". Esta previsión viene a compensar la desigualdad de quienes carecen de recursos que les impiden hacer efectivo el derecho de cumplir con los deberes que la Constitución ordena. Esto es, cuidar efectivamente de la salud.

En el plano internacional el derecho a la salud ha sido reconocido y definido en diversos documentos de alcance universal y regional, tanto americano como europeo. La Declaración Universal de diciembre $1948^{8}$ estableció el derecho a la salud desde la perspectiva inclusiva e integradora al prever que "toda persona tiene derecho a un nivel de vida adecuado que le asegure, así como a su familia, la salud y el bienestar, y en especial, la alimentación, el vestido, la vivienda, la asistencia médica y los servicios necesarios" en el artículo 25. El documento pone énfasis en el valor de la dignidad. En lo regional la Declaración Americana de Derechos y Deberes del Hombre y del Ciudadano de ese mismo año ${ }^{9}$, incorporó en el artículo XI el derecho de toda persona a que su salud sea preservada

5 Puede ampliarse en OMS ONU. "El derecho a la salud". Folleto informativo No. 31. Ginebra, 2008. pp. 15 y 16.

6 Incorporación que se nutre de la influencia de corrientes del derecho comparado que se vieron plasmadas en las primeras décadas del siglo XX, en las Constituciones de Querétaro en México y Weimar en Alemania.

7 CASSINELLI MUÑOZ, Horacio. La salud como derecho humano. En Diccionario Latinoamericano de Bioética. (Juan Carlos Tealdi Coordinador). Editado por UNESCO, RedBioética y la Universidad Nacional de Colombia. Colombia, 2009, pp. 242 y ss.

8 Fue adoptada el 10 de diciembre de 1948 (ONU). Puede ampliarse en BLENGIO VALDÉS, Mariana. "Derechos Humanos en movimiento". Santillana, Montevideo, 2005.

9 Fue adoptada en mayo de 1948 en la XI Conferencia Internacional Americana celebrada en Bogotá junto con la aprobación de la Carta de la OEA. 
por medidas sanitarias y sociales relativas a la alimentación, el vestido, la vivienda y la asistencia médica. ${ }^{10}$

En el año 1966 se adoptan los Pactos Internacionales de Derechos Civiles y Políticos (en adelante PCDP) y Económicos Sociales y Culturales (PDESC) los que conformarán el eje central de protección universal (ONU) desde una perspectiva general en relación a la especificidad de los sujetos de derecho. ${ }^{11}$ El PDESC establece que: "Toda persona tiene derecho al disfrute del más alto nivel posible de salud física y mental” (artículo 12). Como ya hemos visto el Comité creado en virtud de este Pacto ha formulado importantes desarrollos en relación al artículo 12 que consagra el derecho en estudio. Establece además medidas que deben adoptar los Estados para la plena efectividad del derecho que aseguren a todos la asistencia y servicios médicos en caso de enfermedad (inciso d) lo que incluye el acceso a medicamentos.

El reconocimiento y protección del derecho humano a la salud se complementa además con la aprobación de otros tratados que consagran el derecho a la salud de diversa forma y alcance, con aspectos singulares en relación al sujeto titular del derecho y/o las situaciones de especial vulnerabilidad. A modo de ejemplo conviene revisar las previsiones contenidas en la Convención sobre la Eliminación de todas las Formas de Discriminación contra la Mujer (1979) artículo $12^{12}$; la Convención sobre los Derechos del Niño (1989) artículo $24^{13}$; y la Convención sobre los Derechos de las Personas con Discapacidad (2006) artículo $25^{14}$

En el ámbito regional el derecho humano a la salud ha sido objeto de previsiones generales que la comprenden como la del artículo 26 de la Convención Americana sobre Derechos Humanos y otras que la desarrollan como el Protocolo Adicional a la Convención Americana sobre Derechos Humanos en materia de Derechos Económicos Sociales y Culturales (Protocolo de San Salvador). ${ }^{15}$ En el artículo 10 se consagra el derecho de toda persona a la salud, entendida ésta como el disfrute del más alto nivel de bienestar físico, mental y social. Agrega que con el fin de hacerlo efectivo las parte se comprometen a reconocer la salud como un "bien público" y particularmente a adoptar medidas concretas que promuevan la atención integral en toda su extensión. El inciso f) prevé específicamente la satisfacción de las necesidades de salud por los grupos de más alto riesgo y que por sus condiciones de pobreza son los más vulnerables.

10 Estas dos declaraciones son hitos fundamentales para el posterior desarrollo normativo en materia de derechos humanos de la Organización de las Naciones Unidas (ONU) y la Organización de los Estados Americanos (OEA). Con sus agencias y demás órganos especializados como ser la propia Organización Mundial de la Salud (OMS), la Organización Panamericana de la Salud (OPS), UNESCO, UNICEF, ONUSIDA y el Fondo de Población de las Naciones Unidas.

11 El PDCP y su Protocolo Facultativo fueron adoptados el 16 de diciembre de 1966 y ratificados por Uruguay por Ley 13751. En el año 1989 se aprobó el Segundo Protocolo Facultativo referido a la Abolición de la Pena de Muerte el que fuera ratificado por Uruguay por ley 16279. El PDESC fue adoptado el 16 de diciembre de 1966 y su Protocolo Facultativo en 2008. Uruguay ratificó este último por Ley 19029.

12 Uruguay la ratificó por Decreto Ley 15164 de 4 de agosto 1981. Artículo 11.1 f, 12, 14 2) b).

13 Uruguay la ratificó por Ley 16137 de 28 de setiembre 1990. Artículo 24.

14 Uruguay la ratificó por Ley 18418 de 20 de noviembre 2008. Artículo 25.

15 Disponible en: https://www.oas.org/juridico/spanish/tratados/a-52.html Fecha de consulta: 10 agosto 2019. 
Tratados más recientes del sistema regional como la Convención Interamericana sobre la Protección de los Derechos Humanos de las Personas Mayores (2015) ${ }^{16}$ presentan interesantes avances en la dimensión misma del derecho humano a la salud incluyendo una novedosa perspectiva bioética. El texto refiere a problemáticas específicas y amplias. Consagra el derecho de las personas mayores a recibir servicios de cuidado a largo plazo (artículo 12) y el derecho a la salud física y mental sin ningún tipo de discriminación (artículo 19). En forma extensa la previsión enumera aspectos relativos a la atención sanitaria que incluyen la obligación de los Estados de diseñar e implementar políticas públicas inter sectoriales de salud orientadas a la atención integral. Otros vinculados a la necesidad de garantizar el acceso a beneficios y servicios de salud asequibles y de calidad para las personas con enfermedades transmisibles y no transmisibles incluyendo aquellas de trasmisión sexual. Se previó asimismo la obligación de garantizar a la persona mayor la disponibilidad y el acceso a medicamentos reconocidos como esenciales por la OMS incluyendo los necesarios para cuidados paliativos.

El proceso de consolidación de la salud como derecho humano se une intrínsecamente a aspectos esenciales como el daño y el beneficio a la persona, tanto en la dimensión de la clínica como de la investigación. Ese entronque entre el Derecho de los Derechos Humanos y la Bioética se proyecta entre otros factores a partir de revolución que el avance científico y tecnológico produce en la vida, bienestar y salud de la familia humana. Tres declaraciones a nivel universal completan el panorama en relación a los instrumentos reseñados y proporcionan un marco normativo que promueve la "confluencia de pensamientos desde distintos campos y culturales regionales del mundo" en una relación "clara e indivisible con los Derechos Humanos". Ellas son la Declaración Universal del Genoma Humano y Derechos Humanos (1997); la Declaración Internacional sobre Datos Genéticos Humanos (2003) y la Declaración Universal de Bioética y Derechos Humanos (2005) adoptadas todas por la Conferencia General de la UNESCO. ${ }^{17}$

Durante la segunda mitad del siglo XX especialmente el desarrollo científico y los avances bio tecnológicos determinan que las expectativas de vida se alarguen notoriamente. Paulatinamente se instala en la sociedad la idea de la longevidad y la inmortalidad alimentada desde lo intelectual y filosófico por otras disciplinas ${ }^{18}$. Los tratamientos y medicamentos pasan a ser protagónicos en este escenario en el cual las personas exigen atención y servicios, que sean eficaces y de calidad y les aseguren las condiciones para vivir, sobrevivir y morir en condiciones dignas.

\section{OBLIGACIONES DE LOS ESTADOS A PARTIR DE LA NORMATIVA INTERNACIONAL}

El rol del Estado resulta primordial para asegurar el goce de este derecho humano. No ya como un objetivo programático o a largo plazo, sino como una imperiosa necesidad de promover la debida atención sanitaria resolviendo a través de estrategias y políticas

\footnotetext{
${ }^{16}$ Uruguay ratificó la Convención por ley 19430 de 8 setiembre 2016.

17 Pueden verse las tres declaraciones reseñadas y ampliarse en: http://www.unesco.org/new/es/officein-montevideo/social-and-human-sciences/bioethics/ Fecha de consulta: 10 agosto 2019.

18 "El hombre bicentenario" constituye un significativo ejemplo que proyecta y cuestiona la idea de la inmortalidad al dar, su autor, ISAAC ASIMOV, vida a un robot que quiere morir como un ser humano.
} 
públicas los obstáculos o límites que se generan a la hora de disponer de recursos económicos limitados. Así pueden verse los desarrollos jurisprudenciales en elámbitointernacional que invocan el compromiso de los Estados de observar el principio de progresividad en derechos de esta naturaleza con fuerte componente económico y social. A modo de ejemplo la Corte Interamericana de Derechos Humanos ha sostenido en nuevos desarrollos jurisprudenciales, dos tipos de obligaciones que se derivan de los derechos de naturaleza económica y social protegidos en el artículo 26 de la Convención Americana sobre Derechos Humanos. Las obligaciones de "exigibilidad inmediata" y aquellas de "realización progresiva". Al respecto la Corte destaca que: "la obligación de la realización progresiva de los Derechos Económicos Sociales y Culturales (en adelante DESCA) prohíbe la inactividad del Estado en su tarea de implementar acciones para la efectiva protección de estos derechos, sobre todo en aquellas materias donde la ausencia total de protección estatal coloca a las persona ante la inminencia de sufrir un daño a su vida o su integridad personal”. ${ }^{19}$ El órgano jurisdiccional concluye que el artículo 26 de la Convención Americana sobre Derechos Humanos ${ }^{20}$ protege aquellos derechos que derivan de las normas económicas sociales y de educación, ciencia y cultura contenidas en la Carta de la OEA. Los alcances de esos derechos deben ser entendidos en relación con el resto de las demás cláusulas de la Convención Americana sobre Derechos Humanos, por lo que están sujetas a obligaciones generales contenidas en los artículos 1.1 y 1.2 de la Convención y pueden ser sujetos de supervisión por parte de la Corte en términos de los artículos 62 y 63 del mismo instrumento. ${ }^{21} \mathrm{Su}$ fundamentación se basa además de las cuestiones formales, "en la interdependencia e indivisibilidad de los derechos civiles y políticos y los derechos económicos sociales y culturales y ambientales así como su compatibilidad con el objeto y fin de la CADH que es la protección de los derecho fundamentales de los seres humanos".

Por su parte los desarrollos de los órganos internacionales de observancia de los tratados en el ámbito internacional y concretamente los documentos que emanan del Comité de Derechos Económicos Sociales y Culturales (en adelante Comité DESC) permiten proyectar las nuevas dimensiones del derecho humano a la salud. Entre las 24 observaciones generales emitidas a la fecha por este Comité de expertos independientes ${ }^{22}$ destacamos las siguientes: No. 14 "El derecho al disfrute del más alto nivel de salud" ; No. 15 sobre "El derecho al agua"; No. 19 relativa al "Derecho a la seguridad social"; No. 20 que aborda "La no discriminación y los derechos económicos sociales y culturales"; No. 22 sobre "Derecho a la salud sexual y reproductiva" y No. 24 sobre "Obligaciones de los Estado en el contexto de las actividades empresariales".

Según el Comité de DESC en la Observación No. 14 del año 2000 relativa al disfrute del más alto nivel posible de salud constituyen aspectos relevantes en relación a las obligaciones

19 Sentencia Cuscul Pivaral y otros vs. Guatemala. Ver B.4.1.1. (párrafo 141 y ss.) Estándares sobre el derecho a la salud aplicable en relación con el principio de progresividad.

20 Convención Americana sobre Derechos Humanos. Disponible en: https://www.oas.org/dil/esp/ tratados_B-32_Convencion_Americana_sobre_Derechos_Humanos.htm Fecha de consulta: 10 de agosto 2019.

21 Convención Americana sobre Derechos Humanos. Ratificada por Ley 15737 de 8 de marzo 1985. Disponible en: https://www.impo.com.uy/bases/leyes/15737-1985/15 Fecha de consulta: 10 agosto 2019.

22 Disponibles en: https://conf-dts1.unog.ch/1\%20SPA/Tradutek/Derechos_hum_Base/CESCR/ 00_1_obs_grales_Cte\%20Dchos\%20Ec\%20Soc\%20Cult.html Fecha de consulta: 10 agosto 2019. 
estatales: respetar y facilitar el acceso a bienes y servicios de salud; adoptar leyes y otras medidas así como tomar disposiciones apropiadas de carácter legislativo, administrativo, presupuestario y judicial para que sea efectivo el derecho a la salud. ${ }^{23}$

En la última de las observaciones emitidas por el Comité DESC (No. 24), se destaca la necesidad de que se implemente una regulación e intervención directa del Estado en relación a la restricción de publicidad que condicione o afecte el goce de la salud como valor humano. Específicamente observa la necesidad de restringir la comercialización y publicidad de determinados bienes y servicios a fin de "proteger la salud pública" por ejemplo los productos sucedáneos de la leche materna; y los estereotipos de género y discriminación. ${ }^{24}$

\section{CUIDADOS PALIATIVOS.}

Lo anteriormente expresado nos permite reafirmar la existencia de nuevas dimensiones del derecho humano a la salud, las que comprenden aspectos hace décadas inexplorados o no conceptualizados que se desprenden del derecho en sí mismo. Su evolución observa un proceso paulatino de desarrollo por parte de las autoridades sanitarias y un empoderamiento de los usuarios y pacientes. Entre esta nueva dimensión encontramos los "cuidados paliativos", prestaciones reconocidas expresamente en el contexto del derecho humano a la salud, que deben ser proporcionados por los Estados en el marco de sus obligaciones de respeto, protección y promoción de los derechos humanos y específicamente la salud. Los cuidados paliativos ponen su mirada central en la persona y deben proporcionarse a través de servicios de salud integrados.

La Organización Mundial de la Salud señala que los cuidados paliativos constituyen "un enfoque que mejora la calidad de vida de pacientes y sus allegados cuando afrontan problemas de orden físico, sicosocial o espiritual inherentes a una enfermedad potencialmente mortal".$^{25}$ Esta perspectiva apuesta a mejorar la calidad de vida a través de la prevención y alivio del sufrimiento por medio de la identificación lo más temprana posible, el tratamiento del dolor y problemas vinculados a ello tal cual se expresó. Los cuidados paliativos comprenden niños, niñas, adolescentes y adultos. Además de los allegados de los pacientes. Según la OMS "los programas de asistencia paliativa deben utilizar el trabajo en equipo para brindar apoyo a los pacientes y a quienes les proporcionan cuidados. Comprende atención de necesidades prácticas y apoyo sicológico a la hora del duelo". Según la OMS actualmente solo un $14 \%$ de la población mundial que necesita cuidados paliativos la recibe. Se estima que anualmente 40 millones de personas necesitan cuidados paliativos y el $78 \%$ de ellas viven en países de ingreso bajo e ingreso mediano. ${ }^{26}$

${ }^{23}$ Disponible en: http://conf-dts1.unog.ch/1\%20SPA/Tradutek/Derechos_hum_Base/CESCR/ 00_1_obs_grales_Cte\%20Dchos\%20Ec\%20Soc\%20Cult.html\#GEN14 Fecha de consulta: 10 agosto 2019 .

${ }^{24}$ Observación General No. 24. Punto 19. Disponible en: https://conferences.unite.un.org/documentrepositoryindexer/MultiLanguageAlignment.bitext?DocID=238ac05a-066d-4462-83ec0dd2919b20dc\&language1=ENGLISH\&language2=Span Fecha de consulta: 10 agosto 2019.

${ }^{25}$ OMS. Cuidados paliativos. Datos y cifras. Disponible en: https://www.who.int/s/news-room/fact-sheets/ detail/palliative-care Fecha de consulta: 10 agosto 2019.

${ }^{26}$ OMS. Cuidados paliativos. Datos y cifras. Disponible en: https://www.who.int/s/news-room/fact-sheets/ detail/palliative-care Fecha de consulta: 10 agosto 2019 
En relación a la dimensión bioética de los cuidados paliativos, DE SIMONE expresa que las mismas se centran en "el equilibrio apropiado entre luchar por la vida pero aceptando que la muerte es inevitable en el contexto de una enfermedad incurable; a promover la identificación del sufrimiento en sus dimensiones (física, emocional, mental y espiritual) y su alivio, sin necesidad de indicar tratamientos fútiles ni de prolongar artificialmente la agonía, a sostener las demandas que presentan las personas con enfermedades limitantes de la vida y las de su entorno familiar, constituyendo ambos la unidad de tratamiento y a privilegiar las vivencias y valores subjetivos de las personas, antes que promover una imparcialidad objetiva que desnaturaliza la esencia del sufrimiento humano. La valorización de la dimensión subjetiva y la jerarquización de las vivencias de los sujetos constituyen aspectos vinculados a la ética del cuidado". ${ }^{27}$

Los cuidados paliativos no se circunscriben a una única enfermedad como generalmente se visualiza por la población, siendo una prestación no frecuentemente demandada por lo general en relación a los reclamos frecuentes de los usuarios y pacientes. La falta de información en esta temática constituye uno de los impedimentos para la "apropiación por parte de la población de este recurso" y la consolidación efectiva de la prestación. Además de otros obstáculos como la capacidad económica de los centros sanitarios lo que incluye la profesionalización del equipo asistencial.

La OMS identifica que la mayoría de los adultos que necesitan cuidados paliativos padecen enfermedades crónicas como ser: cardiovasculares (38\%), cáncer (34\%), respiratorias crónicas (10.3\%), sida (5.7\%) y diabetes (4.6\%). Otras afecciones como la insuficiencia renal, enfermedades hepáticas crónicas, esclerosis múltiple, enfermedad de Parkinson, artritis reumatoide, enfermedades neurológicas, demencia, anomalías congénitas y tuberculosis resistente a los medicamentos requieren asistencia paliativa. ${ }^{28}$. Los cuidados paliativos también se deben proporcionar a niñas, niños y adolescentes e incluso se prevé la asistencia en fetos y no nacidos en el vínculo con los progenitores y la familia. ${ }^{29}$ Del punto de vista normativo aspectos relacionados con la autonomía progresiva de los niños, niñas y adolescentes resultan relevantes al momento de la adopción de decisiones. ${ }^{30}$

Según la OMS “es el dolor uno de los síntomas más frecuentes y graves experimentados por los pacientes que necesitan cuidados paliativos”. Para lo cual los analgésicos opiáceos

${ }^{27}$ DE SIMONE, Gustavo. Cuidados paliativos. En Diccionario Latinoamericano de Bioética (Tealdi, JC Coordinador). Editado Unesco y Universidad Nacional de Colombia, 2008, pp. 88 y ss.

28 OMS. Cuidados paliativos. Datos y cifras. Disponible en: https://www.who.int/es/news-room/factsheets/detail/palliative-care Fecha de consulta: 1 agosto 2019.

${ }^{29}$ Sobre el punto puede verse BERNADA, Mercedes et al. Abordaje del niño con una enfermedad pasible de cuidados paliativos: Visión desde la Unidad de Cuidados Paliativos del Centro Hospitalario Pereira Rossell. Arch. Pediatr. Urug. [online]. 2010, vol.81, n.4 [cited 2019-08-19], pp.239-247. Los autores abordan aspectos vinculados con la atención de niños y niñas en Hospitales Pereyra Rossell analizándose aspectos tales como identificación y respeto de necesidades, preferencias y valores del niño y la familia; equipo de trabajo; comunicación y toma de decisiones. Disponible en: http:// www.scielo.edu.uy/scielo.php?script=sci_arttext\&pid=S1688-12492010000400004\&lng=en\&nrm=iso ISSN 1688-1249. Fecha de consulta: 1 agosto 2019.

${ }^{30}$ En materia normativa pueden verse la Convención de los Derechos del Niño cuyo artículo 12 expresa el derecho de los niños que están en condiciones de hacerlo a expresar su opinión en todos los asuntos que afectan al mismo. El Código de Niñez y la Adolescencia (ley 17823) reafirma el derecho de niños niñas y adolescentes de acuerdo a la evolución de sus facultades a ser oído y obtener respuestas cuando se tomen decisiones que afecten su vida. 
son esenciales. Según datos de la Organización “el 80 \% de los pacientes con sida o cáncer y el $67 \%$ de los pacientes con enfermedades cardiovasculares o pulmonares obstructivas experimentarán dolor entre moderado e intenso al final de sus vidas. Los opiáceos pueden aliviar síntomas como la dificultad de respirar. Controlar estos síntomas en una etapa temprana es una obligación ética para aliviar el sufrimiento y respetar la dignidad de la persona". ${ }^{31}$

En el ámbito internacional barreras de índole económica alejan los cuidados paliativos de las prestaciones a brindar a la población en general aunque no son los únicos obstáculos indicadores de un acceso insuficiente. La carencia de políticas y sistemas nacionales de asistencia paliativa, la falta de formación de personal sanitario y las dificultades de acceso de la población a opiáceos hace que estos cuidados se transformen en desafíos a materializar en aras de asegurar el efectivo y pleno goce de la salud en casos de patologías crónicas o irreversibles entre otras. Influyen también en la carencia de instrumentación de este tipo de cuidados, obstáculos culturales y sociales y errores de concepto como por ejemplo que solo se habrán de brindar a pacientes con cáncer. Así también los pre conceptos o estereotipos sobre la toxicomanía como una consecuencia del suministro de drogas para el alivio del dolor. Los datos a nivel mundial de carencia en este tipo de prestaciones son relevantes. Varían según los países y el nivel de desarrollo.

\section{V. ¿QUÉ SIGNIFICA PALIAR EL DOLOR?}

Como se deprende de la definición proporcionada la atención sanitaria en este ámbito conlleva asimismo aspectos de carácter espiritual y sicológico del usuario o paciente que debe ser considerado integralmente y también en vinculación con su entorno familiar y social. Los cuidados paliativos están proyectados para mejorar la calidad de vida en todo el curso de la enfermedad y ciclo vital. Los cuidados se destinan a la atención de enfermedades limitantes, crónicas, avanzadas o incurables. Los cuidados paliativos comprenden la atención integral e interdisciplinaria de los pacientes cuya enfermedad no responde a un tratamiento curativo o sufren dolores que pueden ser evitables. Tienen como cometido mejorar la calidad de vida de la persona enferma durante todo el proceso cuya duración es variable, y hasta el final de sus días. En muchos casos implica una atención al control del dolor, de todos los síntomas, además de la preocupación por atender los problemas de relación de tipo emocional, espiritual o familiar de la persona y su entorno. En relación a la etapa final de la vida no se busca retrasarla ni acelerarla sino dejar que la misma siga su curso natural.

Los cuidados paliativos comprenden componentes no solo físicos sino también emocionales que pretenden asegurar al usuario o paciente una atención integral que le asegure el tratamiento total del dolor y le ayuden a lograr calidad de vida durante todo el proceso que dure su enfermedad.

Los principios éticos en cuidados paliativos generalmente aceptados en los Códigos de Etica son los siguientes: principio de inviolabilidad de la vida humana; principio de proporcionalidad terapéutica; principio de doble efecto en el manejo del dolor y la supresión

31 OMS. Cuidados paliativos. Datos y cifras. Disponible en: https://www.who.int/es/news-room/factsheets/detail/palliative-care Fecha de consulta: 1 agosto 2019. 
de la conciencia, principio de prevención y principio de no abandono. Destaca SERNA que el modelo de atención médica que comprende estos principios en materia de cuidados paliativos "contiene un potencial para un profundo cambio en la cultura médica contemporánea, frente a la lógica del recurso tecnológico que con frecuencia nos lleva a considerar que es éticamente justificable o incluso exigible todo lo que es técnicamente posible”. ${ }^{32}$

\section{CONSAGRACIÓN NORMATIVA DE LOS CUIDADOS PALIATIVOS EN URUGUAY.}

La ley 18335 sobre "Derecho y Obligaciones de pacientes y usuarios" aprobada en el año $2008^{33}$ comprende incorpora nuevas dimensiones en relación al derecho humano a la salud y los derechos relativos a la dignidad humana. El artículo 6 establece que toda persona tiene el derecho a acceder a una atención integral que comprenda todas aquellas acciones destinadas a la promoción, protección, recuperación, rehabilitación de la salud y “cuidados paliativos" de acuerdo a las definiciones que establezca el Ministerio de Salud Pública. El artículo 17 prevé que todo paciente tiene derecho a un trato respetuoso y digno, y a procurar que en todos los procedimientos de asistencia médica se evite el dolor físico y emocional de la persona cualquiera sea la situación fisiológica o patológica (inciso B). El artículo comprende otras previsiones relativas a la dignidad en el final de la vida y el respeto de la condición humana en el ámbito sanitario.

Por su parte el Código de Etica Médica del Colegio Médico del Uruguay aprobado por Ley 19286, comprende varias previsiones referidas al tema tanto en la Declaración de Principios Fundamentales como en el capítulo referido al final de la vida. ${ }^{34}$

Los cuidados paliativos constituyen una prestación obligatoria en Uruguay y deben proporcionarse a nivel nacional. Los derechos de los usuarios del Sistema Nacional Integrado de Salud (SNIS) comprenden el "recibir asistencia de calidad humana y especializada a lo largo de todo el ciclo vital, incluso al final de la vida, lo que se denomina "cuidados paliativos". ${ }^{35}$ El Estado uruguayo reconoce el derecho a "recibir un tratamiento efectivo para el dolor en todas sus dimensiones (físico y emocional) y evitarlo siempre que sea posible de cuerdo a la evolución de la patología". Así también a "recibir asistencia de calidad humana y especializada a lo largo de todo el ciclo vital, incluso al final de la vida", reconociendo en ello a los cuidados paliativos. Las pautas que lo rigen son: cobertura universal; asistencia por equipo interdisciplinario; asistencia continua y asistencia de acuerdo al nivel de complejidad.

En el año 2016 Uruguay ratifica la Convención Interamericana sobre Protección de los Derechos Humanos de las Personas Mayores de 2015 (ley 19430) la cual contiene diversas

\footnotetext{
${ }^{32}$ SERNA, Ismael. Principios éticos en cuidados paliativos. En Diccionario Latinoamericano de Bioética. Editado por UNESCO y Universidad Nacional de Colombia. Bogotá. 2008, pp. 91.

${ }^{33}$ Ley 18335 de 2008. Disponible en: https://www.impo.com.uy/bases/leyes/18335-2008 Fecha de consulta: 10 de agosto 2019.

${ }^{34}$ Disponible en: https://legislativo.parlamento.gub.uy/temporales/leytemp889109.htm Fecha de consulta: 10 agosto 2019 .

${ }^{35}$ Conoce tus derechos. MSP. Montevideo, 2019, pp. 13. Disponible en: https://www.gub.uy/ministeriosalud-publica/sites/ministerio-salud-publica/files/documentos/publicaciones/ MSP_DERECHOS_USUARIOS_SALUD.pdf Fecha de consulta: 17 agosto 2019.
} 
previsiones relacionadas con los cuidados paliativos y la dignidad en la muerte a fin de promover la igualdad y no discriminación de todos los mayores de 60 años. Aborda temáticas de especial relevancia como la toma de decisiones en materia de salud, cuidados paliativos y otros aspectos. Esta normativa se suma a la antes relacionada y permite fortalecer esta nueva dimensión de la salud y el tratamiento paliativo.

\section{COBERTURA EN MATERIA DE CUIDADOS PALIATIVOS EN URUGUAY}

En la práctica asistencial los cuidados paliativos existen en el país desde el año 1985 y observan a partir de su obligatoriedad un progresivo aumento que apuesta a superar las dificultades que co existen para brindarlo.

El $36 \%$ iniciaron actividades antes del 2012 en base a iniciativas personales o institucionales. El 64 \% lo hacen una vez que se incorpora como prestación a nivel ministerial ${ }^{36}$.

En noviembre de 2013 el Ministerio de Salud Pública formalizó un Plan Nacional de Cuidados Paliativos con "Guía de buenas prácticas para su implementación" a través del Area de Enfermedades Crónicas No Trasmisibles - DPES -DIGESA. ${ }^{37}$ Se destaca en esta guía: i) la definición de cuidados paliativos: "enfoque que mejora la calidad de vida de pacientes y familias que se enfrentan a problemas asociados con enfermedades amenazantes para la vida, a través de la prevención y alivio del sufrimiento por medio de la identificación temprana e impecable evaluación y tratamiento del dolor y otros problemas, físicos, sicológicos y espirituales". En segundo lugar: ii) los principios generales que hacen a la existencia misma de los cuidados paliativos como su universalidad; atención integral, centrada en el paciente y con enfoque biopsicosocial; accesibilidad y sin costo extra, y recursos humanos especializados. En tercer lugar, iii) los criterios de inclusión a los que nos remitimos expresamente. Estos son según la referida Guía: enfermedad incurable, avanzada y progresiva; respuesta a tratamientos baja o nula; síntomas múltiples; impacto gradual en la autonomía física; intenso impacto emocional en el paciente, familiares y profesionales, pronóstico de vida limitado, dilemas éticos frecuentes relacionados con la nutrición, hidratación, altas y bajas del hospital; nivel alto de necesidades de atención. También se estableció que los niños pasibles de cuidados paliativos son aquellos que: "requieren tratamiento curativo o para prolongar la vida; que requieran tratamiento prolongado para mejorar y mantener la calidad de vida; no tienen opción de tratamiento curativo; o tienen afectación neurológica severa, no progresiva que derivan en vulnerabilidad y complicaciones que pueden causar la muerte prematura". En el Anexo I del Plan se especifican las "Patologías pasibles de recibir asistencia paliativa" (oncología; enfermedad avanzada pulmonar; insuficiencia cardíaca; enfermedad hepática avanzada; insuficiencia renal crónica avanzada; demencias terminales; esclerosis múltiple y lateral amiotrófica; sida en etapa terminal). En el caso de niños los detalla en grupos en la medida que presenten

\footnotetext{
${ }^{36}$ Situación de los cuidados paliativos en Uruguay. Informe observatorio Nacional 2016. Disponible en: file:///C:/Users/Usuario/Downloads/INFORME\%20SITUACION\%20 C U ID AD OS\% 20PALIATIVOS\%202016\%20(2).pdf Fecha de consulta: 10 agosto 2019.

37 Disponible en: file://C:/Users/Usuario/Downloads/GUIA\%20DE\%20BUENAS\%20PRACTICAS\% 20CP_0\%20(1).pdf Fecha de consulta: 1 agosto 2019.
} 
enfermedades o condiciones de salud que amenacen o limiten su vida. En el Anexo II atiende criterios de complejidad. En el III la medicación necesaria para asistencia paliativa; en el IV las competencias profesionales del equipo de cuidados" ${ }^{38}$

Según informe nacional de Cuidados Paliativos de 2017 elaborado por el MSP en el año 2017 se asistieron 8206 pacientes en todo el país con patologías oncológicas y no oncológicas (adultos y niñez) lo que corresponde al 50 \% de la población pasible en recibirlos. Analizado por regiones la cobertura en el norte del país si bien ha aumentado es menor. También es desigual en el sector público y privado. ${ }^{39}$

Entre las asignaturas pendientes para instrumentar esta prestación se encuentran la universalización de la cobertura a mayores y niños a nivel nacional tanto para patologías oncológicas como no oncológicas; la dedicación amplia del personal sanitario incluyendo médicos, enfermería, asistentes sociales, sicólogos a la especialización para asegurar que la misma se brinde en todas las modalidades de asistencia de forma continua. Esto es: internación, domicilio consultorio y guardia telefónica. Los principales obstáculos visualizados en sucesivos informes oficiales se sintetizan en: escasez de recursos humanos; falta de capacitación sobre cuidados paliativos por parte de los profesionales que no derivan a los pacientes; baja carga horaria de los especialistas en cuidados paliativos; falta de formación universitaria en la temática; falta de conocimiento de las autoridades de los cuidados de este tipo lo que determina escaso apoyo. ${ }^{40}$

\section{PROYECCIÓN DE LA TEMÁTICA EN EL SIGLO XXI.}

Las nuevas dimensiones del derecho a la salud comprenden aspectos de especial sensibilidad e importancia a nivel social. Uno de ellos lo constituye el derecho a cuidados paliativos. Como se ha visto su reconocimiento a nivel normativo tiende a consolidarse ya sea en el ámbito legislativo o en vinculación con fuentes internacionales o las propias constituciones. Desarrollos jurisprudenciales en elámbito internacional van en consonancia con este proceso que progresivamente va incrementando una aproximación a la temática acorde con las nuevas exigencias del mundo moderno vinculadas a los procesos asistenciales y a la perspectiva bioética.

Dentro de los obstáculos que emergen de los informes del MSP reseñados ${ }^{41}$, se visualizan las insuficientes oportunidades de formación universitaria o carencias en la formación especializada en la materia. Lo que coincide con las indicaciones que emanan de la OMS en relación a la consolidación de este tipo de cuidados ${ }^{42}$. La capacitación y formación en cuidados paliativos debería incorporarse como estrategia nacional a nivel sanitario y en

\footnotetext{
38 Ver documento anterior, pp. 6 y 7.

${ }^{39}$ Informe 2017 Area Programática Cuidados Paliativos MSP. Disponible en: file://C:/Users/Usuario/ Downloads/MSP_INFORME_NACIONAL_CUIDADOS_PALIATIVOS.pdf Fecha de consulta: 10 agosto 2019.

${ }^{40}$ Situación de los cuidados paliativos en Uruguay. Informe observatorio Nacional 2016. Disponible en: file:///C:/Users/Usuario/Downloads/INFORME\%20SITUACION\%20 CUIDADOS\% 20PALIATIVOS\%202016\%20(2).pdf Fecha de consulta: 10 agosto 2019.

${ }^{41}$ Situación de los cuidados paliativos en Uruguay. Informe observatorio Nacional 2016. Disponible en: file:///C:/Users/Usuario/Downloads/INFORME\%20SITUACION\%20 CUIDADOS\% 20PALIATIVOS\%202016\%20(2).pdf Fecha de consulta: 10 agosto 2019.

42 Acceso insuficiente a cuidados paliativos. Disponible en: https://www.who.int/es/news-room/factsheets/detail/palliative-care Fecha de consulta: 10 agosto 2017.
} 
coordinación con instituciones educativas terciarias a modo de política pública. Así también la previsión de actividades de educación permanente en todas las especialidades médicas y de enfermería como base, sensibilizando al colectivo médico y asistencial en forma amplia e integradora. Lo que incluyen como señalamos diversas profesiones no solo médicas, y las personas que cumplen diversos roles a nivel sanitario, y que en forma imprescindible deben integrar los equipos asistenciales en cuidados paliativos: enfermería, asistentes sociales, sicología, administrativos y voluntarios.

Agregaríamos a esta lista, la necesidad de formación de esta temática en el ámbito jurídico para la comprensión de los operadores del derecho en la función que deban cumplir ya sea como magistrados, defensores, litigantes o asesores, para que conozcan y reconozcan la temática como parte de la dimensión jurídica del derecho humano a la salud y las obligaciones estatales.

Como complemento de la política pública y también como estrategia para eliminar obstáculos cuya identificación también consta en los informes reseñados, sería de especial relevancia la implementación de actividades permanentes y campañas informativas a la población del contenido, importancia y alcance de esta prestación. Quien no conoce la existencia de un derecho difícilmente pueda invocarlo y procurar hacerlo efectivo. Es obligación de los Estados incorporar estrategias de difusión y sensibilidad de la población en materia de derechos humanos y específicamente la salud como un valor a proteger y promover. Una estrategia comunicacional como parte de la política pública en coordinación asimismo con los centros educativos formales y no formales.

La falta de formación o conocimiento de la temática en materia de gestión y específicamente a nivel de autoridades de centros asistenciales, que promuevan o habiliten la consolidación de equipos interdisciplinarios puede constituir otro de los obstáculos a sanear. Así se identifica en informes del MSP del año 2016 y 2017. Su consideración especial facilitará la búsqueda de estrategias que permitan instalar los cuidados paliativos a nivel país y en los diversos centros asistenciales de modo de acortar la brecha entre aquellos que pueden disponer del servicio en el sector privado y el público. Otro elemento a analizar consiste en atribuir a los profesionales la debida carga horaria para que puedan disponer de esta especialidad como eje central de su labor y no compartida en el marco laboral con otras obligaciones. Proporcionando además a nivel institucional los debidos recursos materiales para el desarrollo de la tarea.

Por último y con el mismo objetivo de promover la temática apostando a la eliminación de obstáculos que la enlentecen o frenan, resulta de interés destacar la necesidad de trabajar y promover la discusión de algunos conceptos a nivel jurídico en coordinación con la especialidad en sí para poder identificar el concepto de cuidado paliativo y no confundirlo con otras situaciones asistenciales. Entre algunas previsiones que pueden incorporarse a nivel legislativo se encuentra la necesidad de definir algunos aspectos vinculados al final de la vida y en forma específica a los tipos de sedación utilizados en los cuidados paliativos proporcionados en esta etapa cercana a la muerte. Todo ello con el objetivo de aclarar y desterrar la confusión existente entre la eutanasia activa, comprendida ésta como "la acción u omisión que acelera o causa la muerte del paciente" ${ }^{41}$ y la posibilidad de disminuir la conciencia del paciente para que no experimente sufrimiento al momento de morir.

41 El Código de Etica Médica del Colegio Médico del Uruguay (Ley 19286) establece que la eutanasia activa es "contraria a la ética de la profesión" médica (artículo 46). Señala que en enfermos terminales "es 
Consideramos relevante insistir en que dentro de los grandes temas a debatir en el marco de las nuevas dimensiones del derecho humano a la salud, la integridad física y la vida, se encuentra la "muerte medicamente asistida" como una temática a incorporar en materia de respeto, protección y goce de los derechos humanos. Por lo pronto su debate en Uruguay resulta incipiente. Entendemos que la discusión desde la perspectiva del derecho y la bioética, facilitará la búsqueda de soluciones que proyecten el reconocimiento de la dignidad humana y el efectivo goce de los derechos y libertades inherentes a la persona.

\section{BIBLIOGRAFÍA CONSULTADA.}

BERNADA, Mercedes et al. Abordaje del niño con una enfermedad pasible de cuidados paliativos: Visión desde la Unidad de Cuidados Paliativos del Centro Hospitalario Pereira Rossell. Arch. Pediatr. Urug. [online]. 2010, vol.81, n.4 [cited 2019-08-19], pp.239-247. Disponible en: http:/ /www.scielo.edu.uy/scielo.php?script=sci_arttext\&pid=S1688-12492010000 400004\&lng=en\&nrm=iso ISSN 1688-1249. G

BERRO, Guido. Medicina Legal. Capítulo La muerte. FCU, 2013, pp. 71 y ss.

SERNA, Ismael. Principios éticos en cuidados paliativos. En Diccionario Latinoamericano de Bioética. Editado por UNESCO y Universidad Nacional de Colombia. Bogotá. 2008, pp. 88 y ss.

FARIAS, Gisela, BERTOLINO, Mariela y WENK, Roberto. Cuidados paliativos. Enfermedad y ética. Perspectivas Bioéticas. Año No. 11. Número 20. Primer semestre 2006. Número especial 10 aniversario.

GOMEZ SANCHO, Marcos y OJEDA MARTIN, Manuel. Paliativa Hospital Universitario de Gran Canaria Dr. Negrín. Las Palmas de Gran Canaria. Revisado por Piriz Gabriela. Cuidados paliativos. Control de síntomas. Séptima edición 2014, Montevideo Uruguay. Disponible en: https://www.gub.uy/ministerio-salud-publica/sites/ministerio-salud-publica/files/documentos/publicaciones/Libro\%20CONTROL\%20DE\%20SINTOMAS\% 20EN\%20CUIDADOS \%20PALIATIVOS\%20MINISTERIO\%20DE\%20SALUD.pdf Fecha de consulta: 10 de agosto 2019.

PASTRANA, Tania; DE LIMA, Liliana; WENK, Roberto; EISENCHIAS, Jorge; MONTI, Carolina; ROCAFORT, Javier; CENTENO, Carlos; Atlas de cuidados paliativos en las Américas. Primera edición 2012. Disponible en: https://dadun.unav.edu/bitstream/10171/34465/1/ Atlas\%20de\%20Cuidados\%20Paliativos\%20de\%20Latinoamerica.pdf Fecha de consulta: 10 de agosto 2019.

PECES BARBA, Gregorio. Diez lecciones sobre ética, poder y Derecho. Instituto de DDHH Bartolomé de las Casas. Dykinson SL. Madrid, 2010.

SCHMIDT, Ludwig. Cuidados espirituales. En Diccionario Latinoamericano de Bioética. Editado por UNESCO y Universidad Nacional de Colombia. Bogotá. 2008, pp. 91 y ss.

ZOBOLI, Elma. "Salud integral". En Diccionario Latinoamericano de Bioética. (Juan Carlos Tealdi Coordinador). Editado por UNESCO, RedBioética y la Universidad Nacional de Colombia. Colombia, 2009, pp. 565 y ss.

\footnotetext{
obligación del médico continuar con la asistencia del pacietne con la misma responsabilidad y dedicación, siendo el objetivo de su acción médica, aliviar el sufrimiento físico y moral del paciente, ayudándolo a morir dignamente acorde con sus propios valores. En etapas terminales a la enfermedad no es ético que el médico indique procedimientos diagnósticos o terapéuticos que sean innecesarios y eventualmente perjudiciales para la vida de los pacientes". (artículo 49). Y agrega en el siguiente artículo (50) que el "medico deberá respetar la voluntad de un paciente que libremente ha decidido rechazar los tratamientos que se le indiquen lugo de un adecuado proceso de consentimiento informado".
} 
